Supporting information:

\title{
Fabrication of Large-scale Micro-lens Arrays Based on Screen Printing for Integral Imaging 3D Display
}

Xiongtu Zhou, Yuyan Peng, Rong Peng, Xiangyao Zeng, Yong-ai Zhang, " and Tailiang Guo *

College of Physics and Information Engineering, Fuzhou University, 350002 Fuzhou, Fujian, PR China

*Corresponding authors: Tel: +86 591 87893299, Fax: +86 59187892643

E-mail: yongaizhang@fzu.edu.cn (Y.Z.),gtl_fzu@hotmail.com (T.G.) 


\section{Figure S1:}
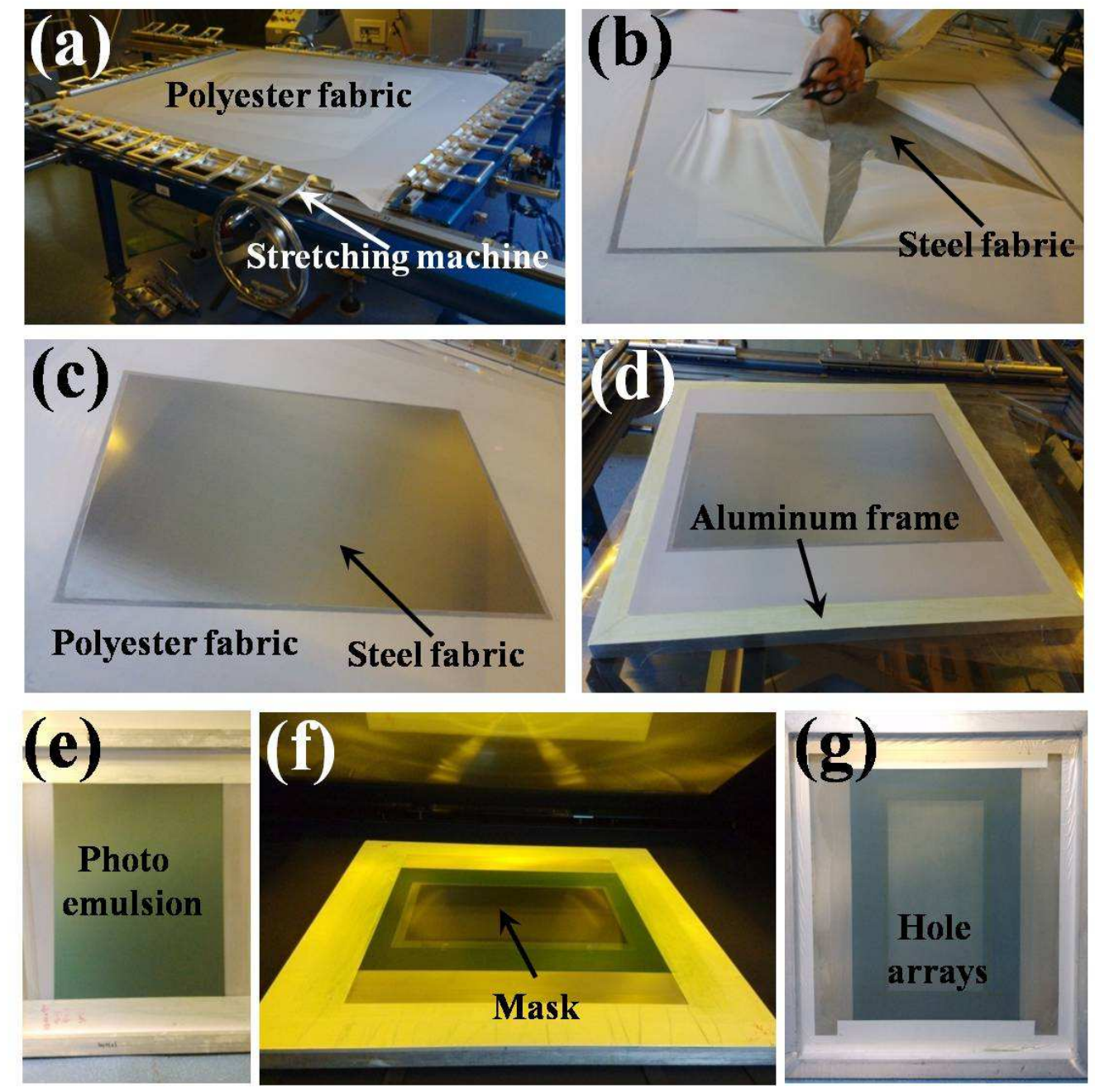

Figure S1 The process flow for the fabrication of screen.

Briefly, the fabrication processes are described as follows:

(1) Finely woven polyester fabric was stretched uniformly using a stretching machine, as shown in Figure S1a.

(2) Finely woven steel fabric was stuck on the center part of the polyester fabric using strength adhesive, then the part of polyester fabric which overlaps with the steel fabric was cut off (Figure S1b), forming the composite fabric (Figure S1c).

(3) An aluminum frame was then stuck on the composite fabric using strength 
adhesive, forming the composite screen (Figure S1d).

(4) Fabrication of mask: The original image (black-and-white negative) was created on a transparent overlay (mask) with a laser printer, as long as the areas to be inked are opaque. The open apertures of screen can be adjusted during the design process of mask.

(5) Photo emulsion (KCS-890, JAPAN) was used as filler material, it was pasted using a squeegee, forming an impermeable substance after UV curing and baking (Figure S1e).

(6) Transfer of patterns to the screen: The mask was placed over the emulsion-coated screen (Figure S1f), and then exposed with UV light of $365 \mathrm{~nm}$. The UV light passes through the transparent areas, leading to a polymerization (hardening) of the emulsion. The areas of emulsion that were not exposed to UV light were dissolved and washed away using deionized water, leaving a negative stencil of the image on the screen (Figure S1g). 
Figure S2:

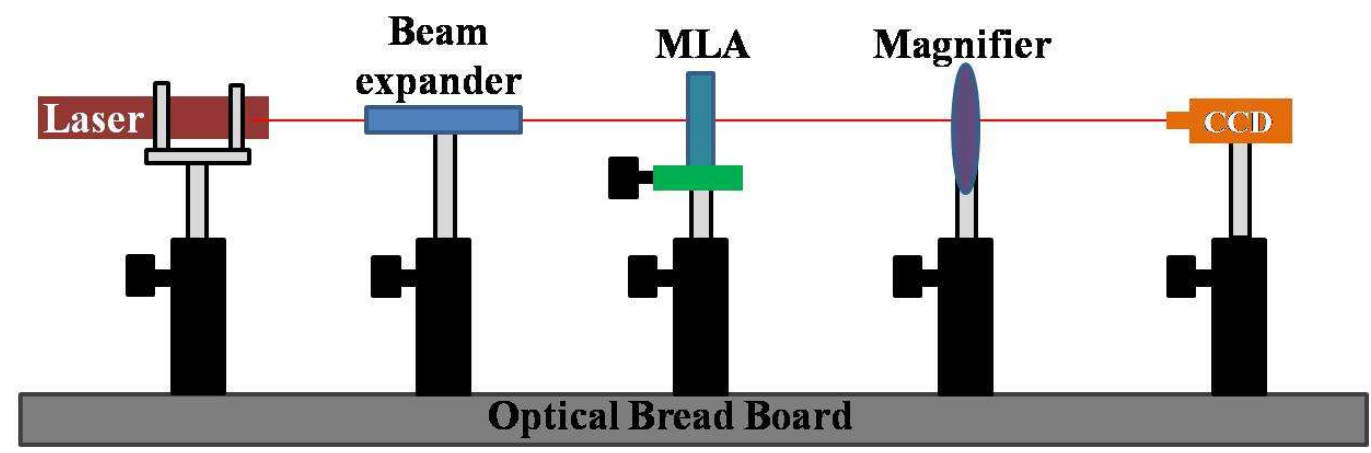

Figure S2 Optical measurement of MLAs. In the optical set-up, laser beam of $650 \mathrm{~nm}$ was expanded and then incident on the microlens array (MLA) vertically, the transmission light was then magnified and was collected by a CCD camera equipped with beam analyzer. The position of MLA was adjusted to capture the sharpest focal point image. The amplification factor was calculated to be 3.28 by dividing the diameter of the lens in the CCD image to the diameter of virtual lens. 


\section{Figure S3:}

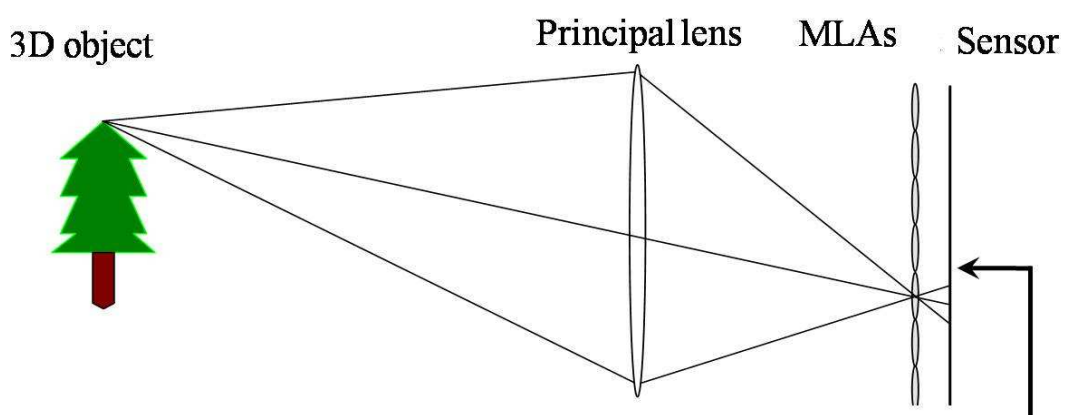

(a) Pick-up of 3D scene using light field camera in Matlab

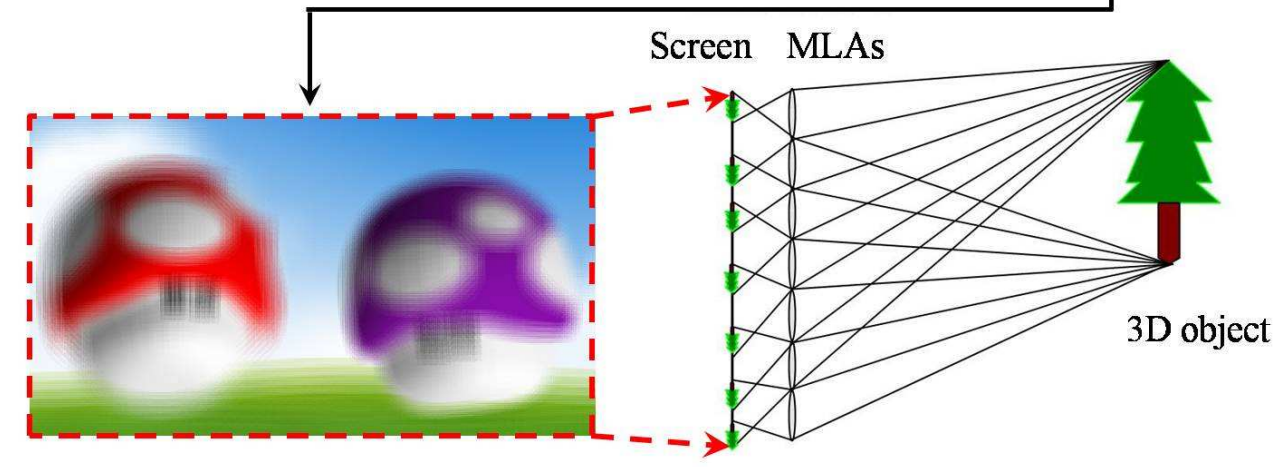

(b) Elemental image arrays

(c) Reconstruction of 3D scene

Figure S3 Illustration of integral imaging 3D display system. (a) The pick-up of elemental image arrays based on computer simulation. In the experiments, the $3 \mathrm{D}$ object was obtained by 3DS MAX modeling software and was saved as an OBJ file, which was then imported in Matlab source codes. The elemental image was then picked up using light field camera, which includes a principal lens and a MLA. The pick-up procedure using light field camera was simulated using computer graphics techniques by tracing the desired rays emanating from the 3D object from different perspectives. An individual elemental image can be recorded by the sensor from a specific perspective. The individual elemental images were then put together to form elemental image arrays, similar to that captured using MLA, which contains the directional information of the 3D object. (b) An elemental image array captured using 
computer graphics techniques. (c) The reconstruction process of $3 \mathrm{D}$ scene. The elemental image arrays were displayed on a screen, which was put behind a virtual MLA fabricated using screen printing, according to the reversibility of optical path, 3D scene can be reproduced. 